\title{
A new type of digital asset: The screencast video - Interview with Al Falaschi and Jake Athey of Widen Enterprises, Inc.
}

\begin{abstract}
Al Falaschi
Audio/Video Product Manager at Widen Enterprises, Inc., leads the product development of audio, video and interactive media handling within Widen's Digital Asset Management (DAM) technologies. Al is also the resident videographer responsible for the production of Widen's audio/video assets for marketing communications and client training.
\end{abstract}

\begin{abstract}
Jake Athey
Marketing Coordinator at Widen Enterprises, Inc., coordinates many of the marketing communications programs for Widen, including the creation and use of interactive media promoting and educating audiences about Widen's services.
\end{abstract}

Keywords: screencast video, digital asset management, audio, video, demo, user interface (UI)

Abstract An interview with Al Falaschi, Audio/Video Product Manager for Widen Enterprises Inc., providing a look into the production and use of screencast videos, particularly screencast demos for the purpose of educating audiences about the functionality and user interface with software applications. Screencast videos are not only a new and emerging type of digital asset, but are also a perfect use case for digital asset management systems to catalog and publish assets to new media channels and social media outlets for maximum use and discoverability.

Journal of Digital Asset Management (2008) 4, 313-317. doi:10.1057/dam.2008.35

\section{WHAT CONSTITUTES A SCREENCAST VIDEO?}

A screencast video, as Widen defines it, must show at least the application window or the entire desktop and the key ingredient - "motion" (at least 8 frames per second) as opposed to still screen captures. Audio must contain either a live narration of actions or a scripted voiceover of what is happening on screen. At Widen, we create two kinds of screencast videos — "live demos" and "scripted demos."

The combination of the audio and the visual shows the functionality (what it does) of the application and the user interface (UI) (how users interact...input and output). Functionality is something that is easily explained using text on a webpage or in a brochure, but UI is not.

Al Falaschi Widen Enterprises, Inc., 6911 Mangrove Ln., Madison, WI 53713, USA Tel: +1 (608) 443-5461 E-mail: afalaschi@widen. com UI is the look and feel of an application. The quality of a UI is judged by each user on how it meets their expectations over time... If I click on this button, then I expect this to happen. The still image of a page may look good to a user, but if the user expects one thing to happen when making a selection, and it doesn't, the final perception of that user is that the UI was clunky or bad. Essentially, video is a set of still images over "time," thus making it a great tool to showcase not only an application's functionality, but the UI as well (Figure 1).

\section{HOW DOES WIDEN USE SCREENCAST VIDEOS?}

Widen uses scripted screencast video primarily on our website for sales and promotional purposes, allowing potential customers to take a quick look (under $2 \mathrm{~min}$ ) at what it does, and how it does it. Screencast videos allow someone in the education phase of the buying cycle to quickly become familiar with our products.

We use live demo screencasts for client training on how to use our digital asset management (DAM) systems. We record initial training sessions with a new customer and populate their DAM system with the video. This 


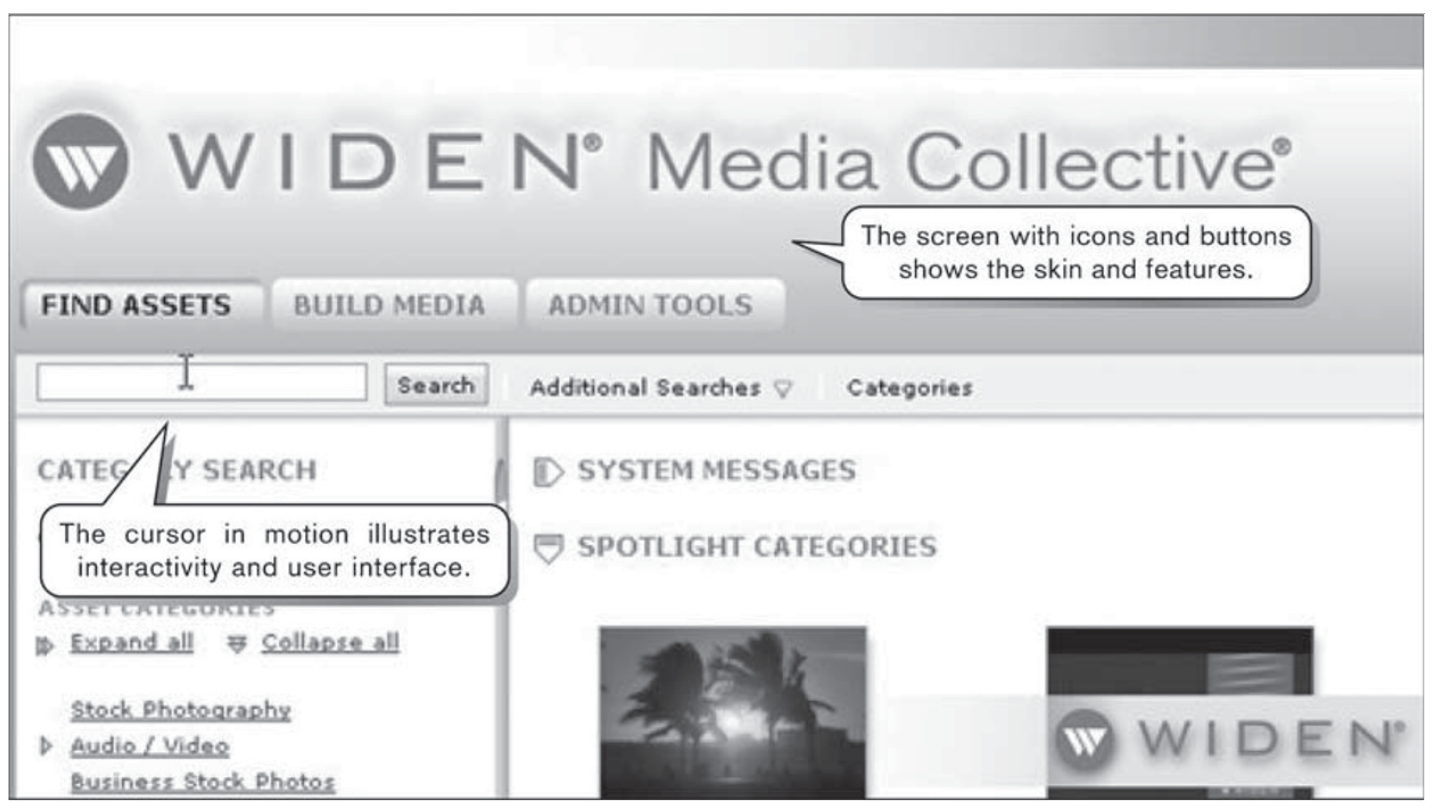

Figure 1: Screen capture of a screencast video from Widen, illustrating the user interface to the Widen Digital Asset Management application

allows new users to view the training videos upon initial login to the DAM system and existing users to take a "refresher course" ondemand. We also use live demos in our color production department, creating training tutorials for our prepress and digital sampling operators.

We use a combination of screencast video and actual video in our research efforts of recording the screen movements and eye movements of new users. This information allows our developers to see what users are doing while using our apps, allowing us to design better UIs that "meet their expectations."

Widen plans to launch a user community that will utilize screencast videos for just-in-time user training. These training videos and tutorials will be available to new users and administrators looking for ways to use more advanced functionality.

\section{HOW DOES WIDEN CREATE SCREENCAST VIDEOS?}

There are a multitude of easy-to-use desktop applications to create screencast video. They range from "free" to a couple of hundred dollars. Some come with software you may already have, like $\mathrm{WebEx}^{\circledR}$ or GoToMeeting ${ }^{\circledR}$. Camtasia Studio is a popular PC-based application and is roughly $\$ 300$. It records screen video and contains editing and audio recording tools.
ScreenFlow has been labeled as the "Camtasia for Mac" and is roughly $\$ 100$. I am a video editor and am more at home in my NLE, so I prefer to use Final Cut Pro and Sony Vegas, which also handles the audio recording.

If you are going for a "live demo" feel, you can simply use the "record audio" function in the screen capture software that you purchased. If you would like a more "scripted" feel, you can record an audio voiceover with a small DAW studio setup. The cheapest and best way to record audio is with a USB powered condenser microphone. This is a studio-quality microphone with a builtin Analog to Digital converter. It converts your voice into a digital signal, which through the USB connection allows you to record into any audio application. These programs range in cost from free (Garage band, Audacity) to the programs I use with price tags up to $\$ 1,500$.

For our "scripted" screencasts, we typically write the voiceover script first. It drives the bus and dictates what is seen on the screen. For "live" screencasts, we typically just write an outline and then start recording. We then record the screen video and audio. Next, I edit the audio, edit the video to match and render a final video file. We then upload the video file to our DAM and publish to our website. Aside from the upfront equipment and software cost, which 
could vary from free to over $\$ 5 \mathrm{k}$, the only cost for doing a video is your time (whatever that is worth). Typically, for a 1-2 minute Widen demo, we concept, write, record, edit and publish in $2-3$ hours.

\section{HOW DOES WIDEN CATALOG THESE ASSETS FOR MAXIMUM USE AND DISCOVERABILITY?}

The cataloging of screencasts as assets is completely dependent upon their use. As I mentioned earlier, Widen uses screencast videos for sales, promotion, training and marketing purposes, which makes DAM the perfect solution. It is the central repository for high definition assets from which they can be repurposed. Widen stores the asset in our DAM system and then orders a Flash Video (.flv) for our website, orders a QuickTime or Windows Media video for download, or orders it authored to a custom DVD and sent to a client. DAM allows you to manage the asset in one place while pushing it to other channels where it will be viewed by your target audience.

\section{HOW DOES WIDEN "PUBLISH" THESE ASSETS?}

We store the video in our DAM in an uncompressed format and convert on the fly to desired formats. All demos are published to our website as flash video (.flv, $720 \times 400,15$ frames per second, at $512 \mathrm{Kbps})$. I may also order a Windows Media file sent to someone on a PC, or have the video authored to a DVD. We also publish to YouTube, which allows others to add it to their website or blog. Since we are primarily a $\mathrm{B}$ to $\mathrm{B}$ company, our visitors are usually on a business connection to the internet. By streaming, the end user will get to see the video almost instantaneously, versus having to wait for the entire file to download. If they do want to download the file, they can order a higher resolution format from our DAM library.

\section{A CHECKLIST FOR IDEATION, CREATION, MANAGEMENT, DISTRIBUTION AND ARCHIVING OF SCREENCAST VIDEOS}

- Most screencast video is used either for training or for demonstration. Decide if you want to "train" someone or if you want to "show'em how it works." Either way, the shorter the better. Keep it under $3 \mathrm{~min}$ if possible.

- Scripting - write a script with two columns video on the left, audio on the right. The video column should be a brief description of what you see on the screen during the block of audio. The audio is simply the voiceover script.

- Record and edit the voiceover with either audio recording or editing software or the audio tools contained in your screencast software.

- Record and edit your screencast to the audio.

- For distribution, you must decide if you want to author files to disc-based media like DVD or if you want it viewed in an online environment. With online video, you must first decide if you want to host video files using your own IT resources or with a third-party service. If it will be viewed often, or by a number of different people at the same time, it will be best to have it hosted by a third party that can handle the bandwidth. Widen offers embed link technology from our DAM system. Video's posted may have multiple preview sizes with html embed codes. By copying and pasting these embed codes, you can add instances of the video to web pages and blogs without having to download and re-upload the video to another system. If you update the video in DAM, it will update everywhere else. Of course there are free options like YouTube, which have embed codes that allow you to add the video to your site while utilizing YouTube's bandwidth. In any case, flash-based video is your best choice, as it has the best penetration rate of any web-based video format. You don't want viewers to have to install any new software to view the files.

- To properly archive, you will need three things: (1) The project file. In the event you use screencast software like Camtasia, you will want to save the project file. If you want to make a minor change to update the video, this file will allow you to quickly reopen your project to change just the desired portion. (2) All of your production files. This includes the unedited version of the video and audio recordings. In order for the project file to open, it needs all of the production files. (3) A high resolution render of the entire video. You store a final, hi-res version of the video in a DAM system. This allows you to quickly search and retrieve the video. Because it is hi res, it will allow you to make quality conversions to other compressed video formats. The DAM system should also help with versioning (Figure 2). 


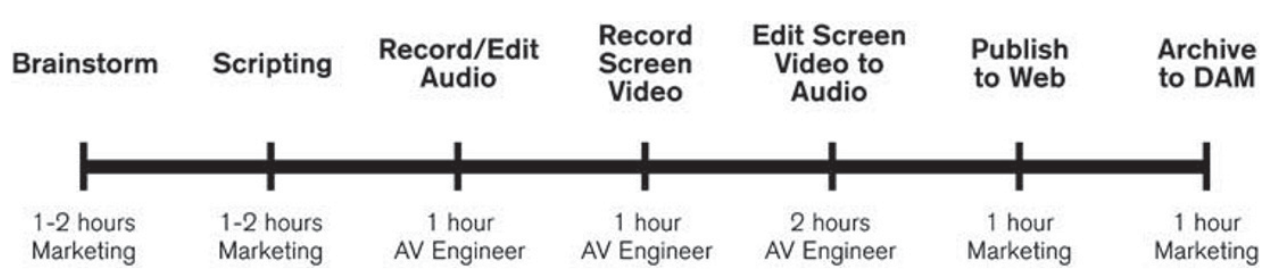

Figure 2: Timeline illustrating the sequence of events (by role) for screencast video creation, publishing and archiving

\section{WHAT DOES THE USER ACTIVITY TELL US REGARDING THE VALUE OF THESE VIDEOS?}

The value proposition of using screencast videos is that it is the most efficient and effective method of communicating the business value of a new or complex process often difficult to visualize by the user. Using a mix of communication methods, screencast videos offer a sense of instant comprehension and instant gratification from the information presented. Video bridges the gap between seller and buyer perspectives, as opposed to the exchange of information in the written and verbal form, which often creates a lot of unknown visual expectations.

As a software marketer, video allows us to articulate more clearly the benefits that the technology can provide. There's no disconnect between what the buyer wants and what the seller is delivering. It forces the visualization of what the buyer thought it might be into something that it will actually be. The reaction from the user base is, "Oh, I get it" in a matter of 60-90 seconds, as opposed to "please clarify" or "more information is needed" as responses to RFPs, whitepapers and datasheets, which take more time to create and consume.

From a marketer's perspective, screencast videos demonstrate a particular audience's need for the product or service, then show how they've satisfied or fulfilled the need accomplishing a great deal of what is needed from "marketing."

\section{WHAT'S THE BUSINESS CASE?}

The business case for using screencast videos is that they allow sales and marketing to better manage customer expectations, which assists with both customer acquisition and customer retention.
On the customer acquisition side, screencast videos are used to shorten the buying cycle because they are used as a means of educating all audiences - from information-gatherers, to influencers and decision-makers.

During education and qualification phases, screencast videos can help key stakeholders visualize the solution at a faster rate and greater level of understanding. As for collaboration and information-sharing phases, videos can be consumed and interpreted much faster than text-based sales tools and with greater impact. Screencast videos convey both the functionality and the user experience, which allow us to better mesh buyer and seller thinking resulting in a higher level of satisfaction because the customer knows exactly what they're getting.

From a personnel standpoint, screencast videos allow salespeople, product managers and technical communications staff to spend less time educating buyers and administrators about the introductory or top-level features and benefits. This allows more time to concentrate on how to leverage the technologies to solve critical business issues for multiple departments. Key decision-makers and stakeholders on the buyer side can devote more attention to specific requirements and the implementation plan.

On the customer retention side, screencast videos are a valuable tool for educating and training the user base about the purpose, functionality and UI of the software solution. Screencast demos are used as one of several methods for educating, training and building strong user adoption during the critical time period following the initial rollout of a new solution. Screencast demos also serve as an excellent source of just-in-time training or quick reference tutorials for new users or those needing a "refresher-course." 


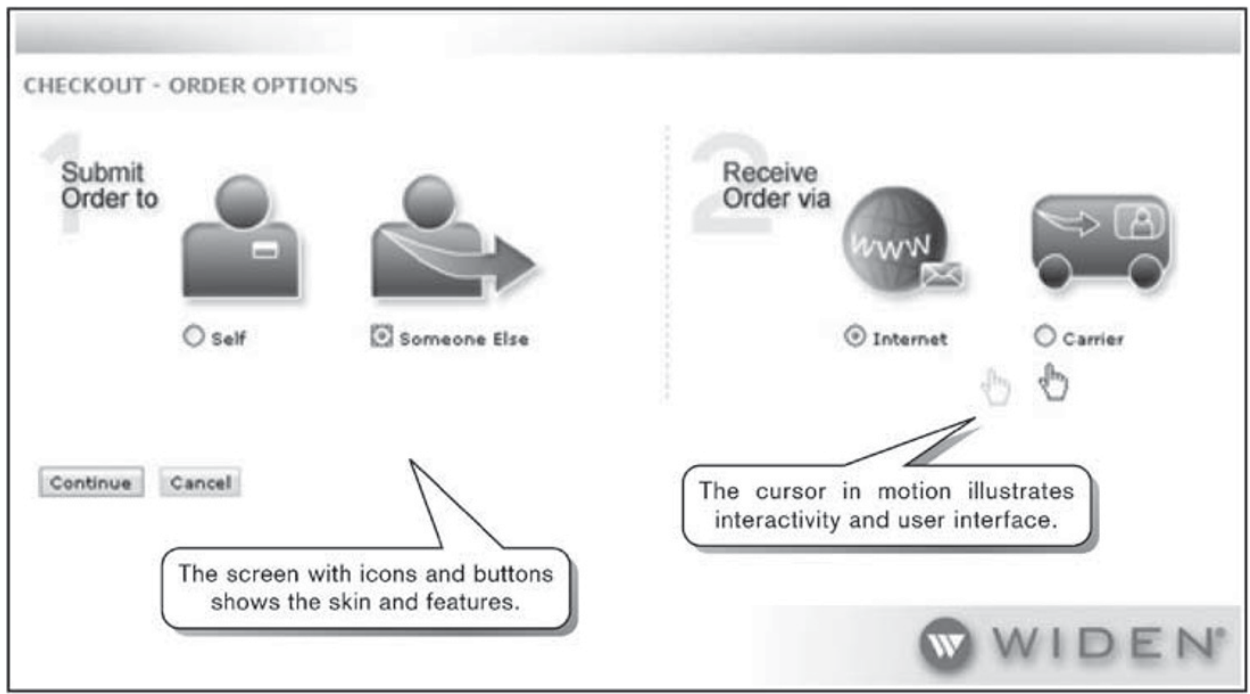

Figure 3: Screen capture of a screencast video from Widen, illustrating the user interface to the Widen Digital Asset Management application. Screencast videos are used to educate prospective customers and to train new users

As a method of building user adoption, the business case of using screencast demos can be made by shortening the time it takes to get users "up-and-running," which, in turn, directly affects ROI and time to ROI.

As for personnel time, screencast videos help alleviate call center volume for both Widen staff and customer administrators. Screencast videos help reduce call volume and troubleshooting from users seeking help with common or simple tasks that may be related to basic functionality or site organization, such has "how do I find this image?" (Figure 3). 